\title{
No difference in the outcome of metastatic thyroid cancer patients when using recombinant or endogenous TSH
}

\author{
Maria Cristina Campopiano ${ }^{1} 1$,*, Debora Podestà 1,*, Francesca Bianchi², Carlotta Giani ${ }^{1}$, Laura Agate ${ }^{1}$, \\ Valeria Bottici', Virginia Cappagli', Loredana Lorusso', Antonio Matrone ${ }^{1}{ }^{1}$, Luciana Puleo', Laura Valerio', \\ David Viola', Paolo Piaggi ${ }^{\left(D^{3}\right.}{ }^{3}$, Rossella Elisei' ${ }^{1}$ and Eleonora Molinaro'
}

${ }^{1}$ Unit of Endocrinology, Department of Clinical and Experimental Medicine, ${ }^{2}$ Department of Nuclear Medicine, University Hospital of Pisa, Pisa, Italy, and ${ }^{3}$ Phoenix Epidemiology and Clinical Research Branch, National Institute of Diabetes and Digestive and Kidney Disease, National Institutes of Health, Phoenix, Arizona

Correspondence

should be addressed

to R Elisei

Email

rossella.elisei@med.unipi.it

\begin{abstract}
Objective: At present, recombinant TSH cannot be used for the treatment of metastatic differentiated thyroid cancer patients. The aim of this study was to evaluate if the type of TSH stimulation, recombinant or endogenous, had an impact on the outcome of these patients.

Design and methods: We compared the outcome of two propensity score-matched groups of metastatic patients, stimulated by either only recombinant TSH $(n=43)$ or only endogenous TSH $(n=34)$.

Results: As expected from the matching procedure, the clinical-pathological features and the cumulative 131-I activities administered to the two groups were very similar. After 4 years of follow-up, $4 \%$ of patients were cured, $3 \%$ had biochemical disease and $93 \%$ had structural disease. However, $91 \%$ of patients obtained a clinical benefit from this therapy in terms of stabilization of the disease or complete remission or partial response. When considering the two groups separately, we did not find any difference in their outcome. When considering the response to 131-I therapy of the single type of metastases, $8 \%$ of lymph node metastases and $8 \%$ of lung metastases disappeared but none of the bone metastases. The response to 131-I therapy of the single type of metastases was similar when we looked at the two groups separately.

Conclusions: This study shows (i) an overall clinical benefit of the 131-I therapy, since the majority of patients remained affected but with a stable disease, and (ii) that the preparation with either recombinant or endogenous TSH has no impact on the 131-I therapy efficacy and the outcome of our two groups of patients.
\end{abstract}

\section{Introduction}

Theinitial therapy of metastatic well-differentiated thyroid cancer (DTC) typically consists of total thyroidectomy, followed by radioiodine (131-I) initial treatment, either for ablation or as adjuvant therapy, and subsequent 131-I radiometabolic treatments in the presence of persistent or recurrent disease (1). The rational basis for the use of 131-I in the diagnosis and therapy of DTC metastases is the ability of follicular cells of well-differentiated primary and metastatic tumors to concentrate iodine. To stimulate the uptake of 131-I by cancer cells, an increase in serum TSH levels is needed and this, nowadays, can be obtained either by withdrawing levo-thyroxine (LT4) therapy, with the consequent increase of endogenous $\mathrm{TSH}$, or by administrating recombinant human TSH (rhTSH),

Published by Bioscientifica Ltd 
an exogenous molecule obtained by recombinant DNA technology $(2,3,4,5)$. The withdrawal of LT-4 therapy induces hypothyroidism that determines consistent negative effects on patients' quality of life (QofL) and social costs $(6,7,8,9)$. Numerous studies already demonstrated rhTSH to be a valid alternative to the LT-4 therapy withdrawal in DTC follow-up and treatment $(10,11,12,13,14)$. Moreover, rhTSH does not induce adverse reactions and antibodies production and allows to preserve euthyroidism and the patient's QofL (15). In DTC patients, who already underwent thyroidectomy, the use of rhTSH is approved for both remnant ablation and follow-up (16). For the radiometabolic treatment of distant metastases, rhTSH can be used only when hypothyroidism could severely harm the patient or in case the endogenous TSH cannot arise for other pathologic reasons $(16,17)$. So far, several retrospective studies have shown that rhTSH is as effective as endogenous TSH in the radiometabolic treatment of metastatic DTC. The majority of these studies have the limit to compare hybrid groups of patients treated with 131-I sometimes after LT-4 withdrawal and sometimes after rhTSH $(11,18$, $19,20)$. Aim of the present study was to compare two groups of metastatic DTC patients treated with 131-I and prepared either by LT- 4 withdrawal or rhTSH at each treatment.

\section{Patients and methods}

\section{Methods}

The study is based on data from 203 medical records of patients with DTC, papillary and follicular histotypes, treated with total thyroidectomy, 131-I remnant ablation and high activities of 131-I for metastatic lesions. Data came from either paper-based clinical records or two digital databases used at the Department of Endocrinology and Metabolism of Pisa University, Italy. An extensive work of information analysis was conducted in this multivariate population to identify two distinct groups of patients differing in the method of 131-I uptake stimulation for the radiometabolic treatment of metastases: 'HYPO group' stimulated by endogenous TSH after LT- 4 withdrawal and 'rhTSH group' stimulated with rhTSH. Of the 203 patients, we first identified 43 patients always administered with rhTSH, then we selected 34 patients characterised by LT- 4 withdrawal by matching for age, sex, staging, American Thyroid Association (ATA) class of risk at the time of surgery.
All patients selected in this preliminary screening had at least one positive post-therapeutic WBS for either lymph node or distant metastases. Moreover, patients belonging to one group were consistently stimulated either by endogenous TSH (HYPO group) or by rhTSH (rhTSH group) whenever subjected to 131-I radiometabolic treatments for metastases.

The overall therapeutic response (outcome) at the end of the follow-up was expressed according to the definition of response to treatment defined by the ATA guidelines (16): (1) structural persistence of disease (SD); (2) biochemical persistence of disease (BD); and (3) clinical remission of disease (CR). To verify the response of the type of metastases, according to the site, in rhTSH and HYPO groups, we considered the different metastases of the same patient separately at the last follow-up. The total number of metastases was 98, 47/98 (48\%) in the lymph nodes, 39/98 (39\%) in the lung and 12/98 (13\%) in the bone.

All patients signed an informed consent for the use of data for research, and the study was approved by the local ethical committee named CEAVNO (Comitato Etico Area Vasta Nord Ovest) of the Azienda OspedalieroUniversitaria Pisana.

\section{Selected study groups}

Seventy-seven out of 203 registered clinical cases fulfilled the inclusion criteria described previously. All patients underwent total thyroidectomy performed by the team of surgeons of the endocrine surgery section of Pisa University in the years between 2001 and 2016. All of them were submitted to an initial 131-I treatment, either for remnant ablation or as adjuvant therapy, and up to three high activity treatments. Patients underwent clinical monitoring between 2003 and 2017 for an average follow-up of 46 months. After surgery, all patients underwent L-T4 therapy at a suppressive dose in order to maintain TSH values $<0.5 \mathrm{mIU} / \mathrm{L}$. The 131 -I activity administered at first treatment for each patient was based on the risk of relapse (1110 MBq vs $3700 \mathrm{MBq}$ ) while for the following treatments we administered a fixed empiric activity of 131-I, according to the site of metastases: 3700 $\mathrm{MBq}$ for lymph node metastases, $4810-5550 \mathrm{MBq}$ for lung metastases and 6660-7400 MBq for bone metastases. The diagnosis of metastatic disease had been made at the time of cancer diagnosis or at the first post-131-I WBS in 45 of 77 patients (58\%). The remaining 32 patients (42\%) developed clinically evident metastatic disease during follow-up. 


\section{Follow-up}

Patients were followed with periodic clinical evaluations every6-12months, includingdosageofserum thyroglobulin (Tg), using a high sensitive chemiluminescent assay (Beckman Coulter, Fullerton, CA, USA); Tg autoantibodies (TgAb), measured by a Fluorescence Enzyme Immuno Assay (AIAPack 2000; Tosoh Corporation, Tokyo, Japan); thyroid hormones free fractions and TSH, dosed by Vitros Immunodiagnostics (Raritan, NJ) and Immulite 2000 (Siemens Healthcare), respectively. Neck ultrasound was performed using a real time instrument (Esaote SPA, My Lab 50 machine with 7.5-12 $\mathrm{MHz}$ linear transducer). In case of suspected lymph node metastases, a fine-needle aspiration was performed for cytological diagnosis and $\mathrm{Tg}$ measurement on the needle washout fluid.

In the presence of a persistent or recurrent disease, patients underwent further radiometabolic treatments with 131-I or other therapies (i.e. surgery, external radiotherapy, other). However, to the purpose of this study, the cases treated with other therapies were excluded to avoid any bias.

Metastatic patients were also submitted to CT scan, and the progression or remission of the disease was calculated according to the response evaluation criteria in solid tumors (RECIST) 1.1 (21).

\section{Statistical analysis}

Statistical analysis was performed by using IBM SPSS Statistics software. Continuous variables were expressed as mean \pm S.D. $(\mathrm{x} \pm$ S.D. $)$ and the categorical variables (nominal and ordinal) presented as a percentage.

The continuous variables analyzed were age; 131-I ablation activity, expressed in $\mathrm{MBq}$; and duration of follow-up. The categorical variables analyzed were gender, age at diagnosis, lymphadenectomy concomitant with thyroid surgical exeresis, extension of lymphadenectomy, histology (differentiating PTC from FTC and aggressive from non-aggressive DTC variants), American Joint Committee on Cancer stage, post-ablation ATA class, mean total activity of administered 131-I and outcome at the end of follow-up.

Student's $t$-test and Pearson's $\chi^{2}$ test were used for the analysis of continuous variables and categorical variables, respectively. Sensitivity analyses were conducted by fitting multinomial logistic regression models, including propensity scores obtained via binary logistic analysis, including clinical variables at baseline as independent variables. A $P<0.05$ was considered as significant.

\section{Results}

The 77 patients were distinguished into two groups: the HYPO group consisting of 34 patients (M: 16; F: 18) and the rhTSH group consisting of 43 patients (M: 21; F: 22).

As shown in Table 1, and as expected on the basis of the selection method, there were no differences in sex distribution, mean age at diagnosis, extension of initial surgery, histology, aggressive variants, stage according to TNM 8th edition (22) and ATA risk stratification (16). The only difference was the greater prevalence of cases with bone metastases in the rhTSH group (Table 2), even if there were no differences in propensity score analysis.

As shown in Table 3, at the end of follow-up, patients of the two groups were treated with the same mean cumulative amount of 131-I activity and the duration of follow-up was comparable. During follow-up, we found $32 / 77$ patients $(42 \%)$ with only lymph node metastases (19/43 (44\%) in the rhTSH group and 13/34 (38\%) in the HYPO group); $18 / 77$ patients (23\%) with lung metastases (9/43 (21\%) in the rhTSH group and 9/34 (27\%) in the HYPO group); 15/77 patients (19\%) with lung and lymph node metastases $(5 / 43(12 \%)$ in the rhTSH group and $10 / 34$ (29\%) in the HYPO group); $5 / 77$ patients (7\%) had bone metastases (4/43 (9\%) in the rhTSH group and 1/34 (3\%) in the HYPO group); we also had $1 / 77$ patients (1\%) with both bone and lymph node metastases, included in the HYPO group (1/34 (3\%)), and 6/77 patients (8\%) with both lung and bone metastases and all are included in rhTSH group (6/43 (14\%)). The statistical analysis did not show any statistically significant difference even if patients with both lung and bone metastases were all included in the rhTSH group and patients with bone metastases were more prevalent in rhTSH group than HYPO group (10/43 (23\%) vs $2 / 34(6 \%))$.

When we looked at the outcome of the patients at the end of follow-up (mean 46 months) (Fig. 1, panel A), as first result we found that only $4 \%$ of overall patients were cured, 3\% had a biochemical disease and 93\% had a persistent structural disease. Moreover, we observed that the distribution of clinical remission, biochemical disease and structural disease cases was similar in both rhTSH group and HYPO group of patients (Fig. 1, panel B). As shown in Table 4, when we looked at the site of metastases at the end of follow-up, we found that the distribution was similar in the HYPO group and in the rhTSH group.

When considering the response to 131-I therapy of the single type of metastases (i.e. no longer detectable at any imaging), 8\% of lymph node metastases and $8 \%$ of lung metastases responded (R) but none of the bone 
Table 1 Epidemiological, clinical, and pathological characteristics of the enrolled patients.

\begin{tabular}{l}
\hline Parameters \\
\hline Females $(n, \%)$ \\
Age (years); mean \pm S.D. \\
Minimum \\
Maximum \\
Total thyroidectomy $(n(\%))$ \\
Lymphnode dissection $(n(\%))$ \\
CC \\
CC + LC \\
LC \\
Histology $(n(\%))$ \\
PTC \\
FTC \\
Aggressive variants $(n(\%))$ \\
AJCC stage $(n(\%))$ \\
I-II \\
III-IV \\
ATA risk stratification post RAI $(n(\%))$ \\
High \\
Intermediate \\
Low
\end{tabular}

\begin{tabular}{c}
\hline Study group $(n=77)$ \\
\hline $40(52 \%)$ \\
$45 \pm 19$ \\
12 \\
80 \\
$77(100 \%)$ \\
$37(48 \%)$ \\
$5(14 \%)$ \\
$25(67 \%)$ \\
$7(19 \%)$ \\
$64(83 \%)$ \\
$13(17 \%)$ \\
$15(20 \%)$ \\
$37(48 \%)$ \\
$40(52 \%)$ \\
$36(47 \%)$ \\
$34(44 \%)$ \\
$7(9 \%)$
\end{tabular}

\begin{tabular}{c}
\hline rhTSH group $(n=43)$ \\
\hline $22(51 \%)$ \\
$48 \pm 20$ \\
\\
$43(100 \%)$ \\
$21(49 \%)$ \\
$4(19 \%)$ \\
$15(71 \%)$ \\
$2(10 \%)$ \\
$35(91 \%)$ \\
$8(9 \%)$ \\
$7(16 \%)$ \\
$18(42 \%)$ \\
$25(58 \%)$ \\
$23(54 \%)$ \\
$19(44 \%)$ \\
$1(2 \%)$
\end{tabular}

\begin{tabular}{clc}
\hline HYPO group $(n=34)$ & & $\boldsymbol{P}$ \\
$\begin{array}{c}18(53 \%) \\
41 \pm 17\end{array}$ & & N.S. \\
N.S.
\end{tabular}

AJCC, American Joint Committee on Cancer; ATA, American Thyroid Association; CC, central compartment; FTC, follicular thyroid cancer; LC, latero-cervical compartment; PTC, papillary thyroid cancer; RAl, radioiodine therapy.

metastases (NR). The response to 131-I therapy was similar when considering the single site of metastases (i.e. lymph nodes, lung and bone) in the two groups of DTC patients (Fig. 2).

When we evaluated the response to RAI treatment according to RECIST (21) we found that, at the end of follow up, 64/77 (83\%) patients showed a stable disease (SD), 5/77 (6.5\%) had a complete remission of the structural disease (CR), 1/77 (1.5\%) had a partial response (PR) and 7/77 (9.0\%) had a progressive disease (PD). Overall, 91\% of patients obtained a clinical benefit from this therapy in terms of stabilization of the disease or complete remission or partial response. When we did this analysis in the two groups separately we found that in the rhTSH group 35/43 (82\%) patients showed a SD, 1/43 (2\%) had a CR of the structural disease, 1/43 (2\%) had a PR and 6/43 (14\%) had a PD while in the HYPO group 29/34 (85\%) patients showed a SD, 4/34 (12\%) had a CR, none

Table 2 Sites of metastases at the first WBS detection in the two groups of patients (rhTSH vs HYPO). Data are presented as $n(\%)$.

\begin{tabular}{|c|c|c|c|c|}
\hline $\begin{array}{l}\text { Sites of } \\
\text { metastases }\end{array}$ & $\begin{array}{c}\text { Study group } \\
\quad(n=77)\end{array}$ & $\begin{array}{c}\text { rhTSH group } \\
(n=43)\end{array}$ & $\begin{array}{c}\text { HYPO group } \\
(n=34)\end{array}$ & $\mathbf{P}$ \\
\hline Lymph node & 18/77 (23\%) & $12 / 18(66 \%)$ & $6 / 18(34 \%)$ & N.S. \\
\hline Lung & 26/77 (34\%) & $17 / 26(65 \%)$ & $9 / 26(35 \%)$ & N.S. \\
\hline Bone & $11 / 77(14 \%)$ & $10 / 11(90 \%)$ & $1 / 11(10 \%)$ & N.S. \\
\hline
\end{tabular}

had a PR and 1/34 (3\%) had a PD. The statistical analysis did not show any significant difference $(P=0.1)$ although a greater percentage of $\mathrm{CR}$ and a lower percentage of PD was observed in the HYPO group. It is worth to note that the CR patients had sub-centimetric lung lesions $(3 / 5(60 \%))$ or lymph node metastases $(2 / 5(40 \%))$. At variance, the patients with PD had bone metastases in $3 / 7$ cases $(44 \%)$, both lung and bone metastases in 1 case (14\%), lung metastases in $2 / 7$ cases (28\%) and lymph node metastases in 1 case $(14 \%)$. Focusing on PD in the two groups separately, we found no significant difference, also after propensity score analysis (data not shown).

\section{Discussion}

Radioiodine therapy is the gold standard therapy for metastatic DTC and 131-I treatments can be repeated until the evidence of either clinical remission or clinical benefit (1). To maximize the therapeutic effect of 131-I, a TSH stimulation of the metastatic cells is required to increase their ability to take up 131-I. So far, the use of rhTSH has been approved for both diagnostic purposes (i.e. Tg stimulation) and thyroid remnant ablation but not for the treatment of metastatic diseases. One of the reasons for the lack of approval of rhTSH for the treatment of metastatic disease is the absence of a prospective and randomized study showing the non-inferiority of the two techniques. Several retrospective studies are available, but most of 
Table 3 Therapeutic and follow-up characteristics of the enrolled patients.

\begin{tabular}{l}
\hline Parameters \\
\hline Cumulative 131-I activity (MBq); mean \pm S.D. \\
Follow-up (months) \\
Mean \pm S.D. \\
Minimum \\
Maximum \\
Median
\end{tabular}

\begin{tabular}{c} 
Study group $(n=77)$ \\
\hline $14800 \pm 4773$ \\
$46 \pm 39$ \\
10 \\
262 \\
37
\end{tabular}

\begin{tabular}{ccc}
\hline HYPO group $(n=34)$ & & $\frac{P}{15725 \pm 4255}$ \\
$48 \pm 27$ & & N.S. \\
39 & & \\
& & \\
\hline
\end{tabular}

them are criticized not only for the retrospective nature but also because the same patient was treated sometimes with rhTSH and sometimes with hypothyroidism $(19,20)$.

Although our study is retrospective, we made an extensive selection to find patients who were prepared only with rhTSH or only with LT-4 withdrawal during
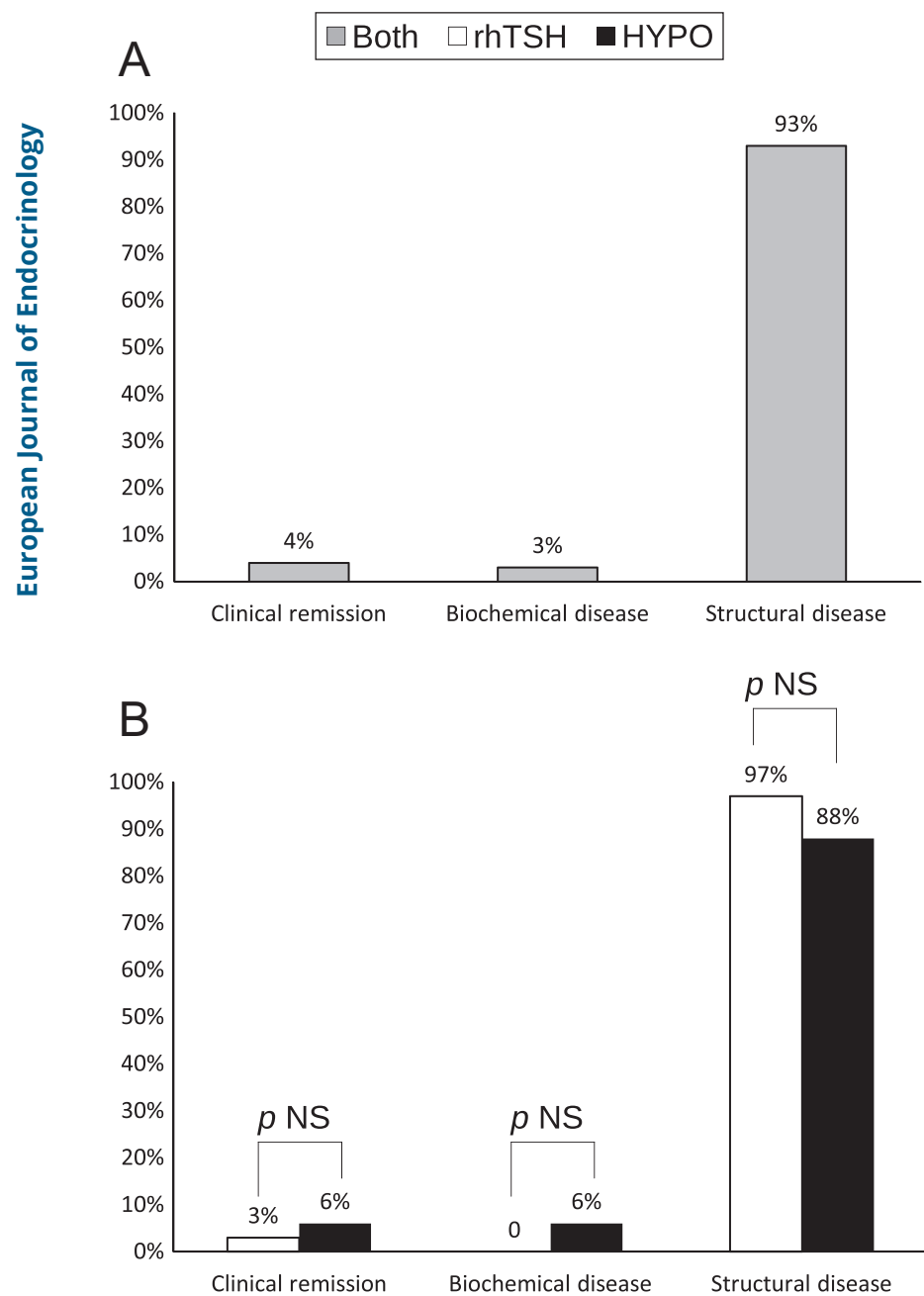

\section{Figure 1}

Panel A: Outcome at last follow-up of overall patients (rhTSH and HYPO). Panel B: Outcome at last follow-up of patients distinguished in the two groups (rhTSH vs HYPO). their follow-up. Moreover, we matched the patients for their epidemiological and clinical features to have two comparable groups of patients as we could expect to have if they were randomized at the time of the first 131-I treatment. The comparison of these two groups of patients showed that their outcome was similar after a median follow-up of about 4 years. The distribution of metastases was also similar between the two groups at the last follow-up even if bone metastases were more prevalent in rhTSH group. This difference reflects the initial unequal and fortuitous distribution of metastases that represents a limitation of this study not easy to be overcome even by an initial randomization, since many of these lesions were detected after the first 131-I treatment. Nevertheless, our study clearly showed that the two types of preparation have no impact on the response to 131-I according to the site of lesions.

Some authors are against the use of rhTSH for the treatment of metastatic lesions because of a lower retention time of 131-I in the tumoral cells (23). So far, nobody has demonstrated that a greater 131-I retention time improves the response to 131-I and the outcome. In our opinion, the fact that there was no difference in the outcome of our patients is indirectly a proof of the fact that the 131-I retention time in the metastatic lesions should not influence the effectiveness of the 131-I therapy.

Finally, we found that only a minority of metastatic patients were in clinical remission, while the bigger majority still showed a structural disease after several treatments with 131-I. This is in line with other studies showing that only $1 / 3$ of metastases are definitively

Table 4 Sites of metastases at the last WBS detection in the two groups of patients (rhTSH vs HYPO). Data are presented as $n(\%)$.

\begin{tabular}{|c|c|c|c|c|}
\hline $\begin{array}{l}\text { Sites of } \\
\text { metastases }\end{array}$ & $\begin{array}{c}\text { Study group } \\
(n=77)\end{array}$ & $\begin{array}{c}\text { rhTSH group } \\
(n=43)\end{array}$ & $\begin{array}{c}\text { HYPO group } \\
(n=34)\end{array}$ & $\boldsymbol{P}$ \\
\hline $\begin{array}{l}\text { Lymph } \\
\text { node }\end{array}$ & $44 / 77$ (57\%) & $22 / 44(50 \%)$ & $22 / 44(50 \%)$ & N.S. \\
\hline Lung & $36 / 77$ (47\%) & $20 / 36$ (56\%) & $10 / 36(44 \%)$ & N.S. \\
\hline Bone & $12 / 77(16 \%)$ & $10 / 12(83 \%)$ & $2 / 12(17 \%)$ & N.S. \\
\hline
\end{tabular}




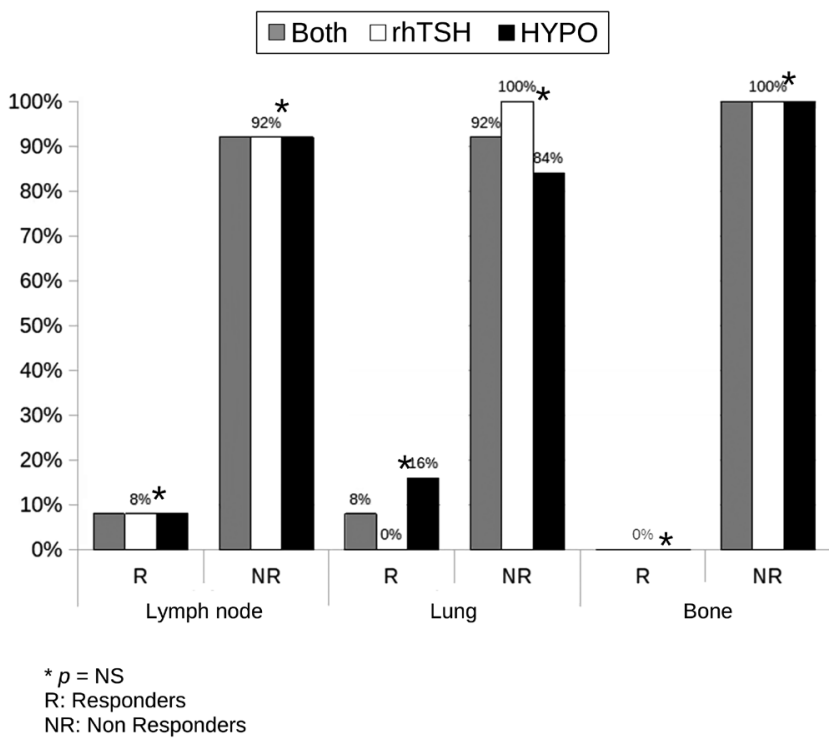

\section{Figure 2}

Response to 131-I therapy according to metastases' site distribution.

cured with 131-I (24), questioning the real curative role of 131-I in metastatic DTC patients. Anyway, our results about the response to RAI treatment according to RECIST (21) allowed us to appreciate the clinical benefit of 131-I therapy (91\% of patients) either in slowing down the disease progression or determining its stabilization. We are conscious that our study has a few limitations: (i) the low number of patients; (ii) the retrospective nature of the study; (iii) the absence of randomization; and (iv) the greater number of bone metastases in the rhTSH group. It is known that metastatic DTC, especially those with distant metastases, are rare $(<5-10 \%)$ : we collected 203 patients out of about 4000 cases followed in our institution in the frame time of the selection (15 years). Moreover, the number of cases prepared with rhTSH at any treatment for 'compassionate use' was even smaller. On average, only $<1 \%$ of cases treated in our Hospital in 1 year are prepared with rhTSH as 'compassionate use' and this is in line with the number of cases that we found $(n=43)$. Of course, we could not further reduce this number by reducing the number of cases with bone lesions. At the same time, we did not find an equivalent number of cases with bone lesions and prepared with LT- 4 withdrawal at any treatment. Thus, by matching the cases, we obtained to avoid statistical differences although it is evident that, in general, a greater prevalence of advanced cases was present in the rhTSH group (i.e. higher prevalence of advanced stages, higher prevalence of high-risk cases, higher prevalence of bone lesions).
The retrospective nature of the study is an unsurmountable limit since a prospective study is impossible if not performed as a multicentric and international study whose costs would be very high and difficult to be sustained by academic institutions. Nevertheless, the higher prevalence of advanced cases in the rhTSH group should negatively weigh on the results of the study while the study, despite these limitations, demonstrates a similar outcome of patients independently from the type of preparation.

In conclusion, our study shows (i) an overall clinical benefit of the 131-I therapy, since the majority of patients remained affected but with a stable disease and (ii) that the preparation with either rhTSH or hypothyroidism has no impact on the efficacy of 131-I therapy. Moreover, we showed that there was no difference in the outcome of our patients and also in the response to 131-I treatment according to the site of metastatic lesions. Since hypothyroidism can affect the QofL of these patients, which is really compromised by the disease itself, our results indicate that rhTSH should be preferred anytime the clinicians and the patients would prefer.

\section{Declaration of interest}

$\mathrm{R} E$ is a consultant for Sanofi-Genzyme. Rossella Elisei is on the editorial board of Reproduction. Rossella Elisei was not involved in the review or editorial process for this paper, on which she is listed as an author. All the other co-authors have no potential conflicts of interest.

\section{Funding}

This research did not receive any specific grant from any funding agency in the public, commercial or not-for-profit sector.

\section{References}

1 Haugen BR, Alexander EK, Bible KC, Doherty GM, Mandel SJ, Nikiforov YE, Pacini F, Randolph GW, Sawka AM, Schlumberger M et al. 2015 American Thyroid Association management guidelines for adult patients with thyroid nodules and differentiated thyroid cancer: the American Thyroid Association Guidelines Task Force on thyroid nodules and differentiated thyroid cancer. Thyroid 201626 1-133. (https://doi.org/10.1089/thy.2015.0020)

2 Pacini F, Ladenson PW, Schlumberger M, Driedger A, Luster M, Kloos RT, Sherman S, Haugen B, Corone C, Molinaro E et al. Radioiodine ablation of thyroid remnants after preparation with recombinant human thyrotropin in differentiated thyroid carcinoma: results of an international, randomized, controlled study. Journal of Clinical Endocrinology and Metabolism 200691 926-932. (https://doi.org/10.1210/jc.2005-1651)

3 Robbins RJ, Larson SM, Sinha N, Shaha A, Divgi C, Pentlow KS, Ghossein R \& Tuttle RM. A retrospective review of the effectiveness of recombinant human TSH as a preparation for radioiodine thyroid remnant ablation. Journal of Nuclear Medicine 200243 $1482-1488$. 
4 Robbins RJ, Tuttle RM, Sonenberg M, Shaha A, Sharaf R, Robbins H, Fleisher M \& Larson SM. Radioiodine ablation of thyroid remnants after preparation with recombinant human thyrotropin. Thyroid 200111 865-869. (https://doi.org/10.1089/105072501316973127) 5 Barbaro D, Boni G, Meucci G, Simi U, Lapi P, Orsini P, Pasquini C, Turco A \& Mariani G. Recombinant human thyroid-stimulating hormone is effective for radioiodine ablation of post-surgical thyroid remnants. Nuclear Medicine Communications 200627 627-632. (https://doi.org/10.1097/00006231-200608000-00005)

6 Badihian S, Jalalpour P, Mirdamadi M \& Moslehi M. Quality of life, anxiety and depression in patients with differentiated thyroid cancer under short term hypothyroidism induced by levothyroxine withdrawal. Klinicka Onkologie 29 439-444. (https:// doi.org/10.14735/amko2016439)

7 Duntas LH \& Biondi B. Short-term hypothyroidism after levothyroxine-withdrawal in patients with differentiated thyroid cancer: clinical and quality of life consequences. European Journal of Endocrinology 2007156 13-19. (https://doi.org/10.1530/eje.1.02310)

8 Dow KH, Ferrell BR \& Anello C. Quality-of-life changes in patients with thyroid cancer after withdrawal of thyroid hormone therapy. Thyroid 19977 613-619. (https://doi.org/10.1089/thy.1997.7.613)

9 An JH, Song KH, Kim DL \& Kim SK. Effects of thyroid hormone withdrawal on metabolic and cardiovascular parameters during radioactive iodine therapy in differentiated thyroid cancer. Journal of International Medical Research 201745 38-50. (https://doi. org/10.1177/0300060516664242)

10 Wang TS, Cheung K, Mehta P, Roman SA, Walker HD \& Sosa JA. To stimulate or withdraw? A cost-utility analysis of recombinant human thyrotropin versus thyroxine withdrawal for radioiodine ablation in patients with low-risk differentiated thyroid cancer in the United States. Journal of Clinical Endocrinology and Metabolism 201095 1672-1680. (https://doi.org/10.1210/jc.2009-1803)

11 Luster M, Felbinger R, Dietlein M \& Reiners C. Thyroid hormone withdrawal in patients with differentiated thyroid carcinoma: a one hundred thirty-patient pilot survey on consequences of hypothyroidism and a pharmacoeconomic comparison to Recombinant Thyrotropin Administration. Thyroid 200515 1147-1155. (https://doi.org/10.1089/thy.2005.15.1147)

12 Borger J, Remy H, Chevalier J, Ricard M, Allyn M, Schlumberger \& De Pourvouville G. Length and cost of hospital stay of radioiodine ablation in thyroid cancer patients: comparison between preparation with thyroid hormone withdrawal and thyrogen. European Journal of Nuclear Medicine and Molecular Imaging 200835 1457-1463. (https:// doi.org/10.1007/s00259-008-0754-9)

13 Guo Y, Zhang Y, Chen Z \& Xin Z. Effects of recombinant human thyroid stimulating hormone on 131I therapy for the treatment of differentiated thyroid cancer. Experimental and Therapeutic Medicine 20159 1847-1850. (https://doi.org/10.3892/etm.2015.2330)

14 Lee J, Yun MJ, Nam KH, Chung WY, Soh EY \& Park CS. Quality of life and effectiveness comparisons of thyroxine withdrawal, triiodothyronine withdrawal, and recombinant thyroid-stimulating hormone administration for low-dose radioiodine remnant ablation of differentiated thyroid carcinoma. Thyroid 201020 173-179. (https://doi.org/10.1089/thy.2009.0187)
15 Testori O, Bagnasco M, Banti E, Bombardieri E, Dottorini ME, Fugazzola L, Maffioli L, Perotti G, Rubello D \& Seregni E. Radiometabolic therapy for metastatic thyroid carcinoma: overview of the literature and rational bases for the use of recombinant human TSH. Minerva Medica 2012103 209-218.

16 Haugen BR, Sawka AM, Alexander EK, Bible KC, Caturegli P, Doherty GM, Mandel SJ, Morris JC, Nassar A, Pacini F et al. American Thyroid Association guidelines on the management of thyroid nodules and differentiated thyroid cancer task force review and recommendation on the proposed renaming of encapsulated follicular variant papillary thyroid carcinoma without invasion to noninvasive follicular thyroid neoplasm with papillary-like nuclear features. Thyroid 201727 481-483. (https://doi.org/10.1089/ thy.2016.0628)

17 Robbins RJ, Driedger A, Magner J \& U.S. and Canadian Thyrogen Compassionate Use Program Investigator Group. Recombinant human thyrotropin-assisted radioiodine therapy for patients with metastatic thyroid cancer who could not elevate endogenous thyrotropin or be withdrawn from thyroxine. Thyroid 200616 1121-1130. (https://doi.org/10.1089/thy.2006.16.1121)

18 Luster M, Lippi F, Jarzab B, Perros P, Lassmann M, Reiners C \& Pacini F. rhTSH-aided radioiodine ablation and treatment of differentiated thyroid carcinoma: a comprehensive review. EndocrineRelated Cancer 200512 49-64. (https://doi.org/10.1677/erc.1.00830)

19 Tala H, Robbins R, Fagin JA, Larson SM \& Tuttle RM. Five-year survival is similar in thyroid cancer patients with distant metastases prepared for radioactive iodine therapy with either thyroid hormone withdrawal or recombinant human TSH. Journal of Clinical Endocrinology and Metabolism 201196 2105-2111. (https://doi. org/10.1210/jc.2011-0305)

20 Klubo-Gwiezdzinska J, Burman KD, Van Nostrand D, Mete M, Jonklaas J \& Wartofsky L. Radioiodine treatment of metastatic thyroid cancer: relative efficacy and side effect profile of preparation by thyroid hormone withdrawal versus recombinant human thyrotropin. Thyroid 201222 310-317. (https://doi.org/10.1089/thy.2011.0235)

21 Eisenhauer EA, Therasse P, Bogaerts J, Schwartz LH, Sargent D, Ford R, Dancey J, Arbuck S, Gwyther S, Mooney M et al. New response evaluation criteria in solid tumours: revised RECIST guideline (version 1.1). European Journal of Cancer 200945 228-247. (https://doi.org/10.1016/j.ejca.2008.10.026)

22 AJCC - American Joint Committee on Cancer. (available at: https:// cancerstaging.org/Pages/default.aspx). Accessed on 30 January 2020.

23 Pötzi C, Moameni A, Karanikas G, Preitfellner J, Becherer A, Pirich C \& Dudczak R. Comparison of iodine uptake in tumour and nontumour tissue under thyroid hormone deprivation and with recombinant human thyrotropin in thyroid cancer patients. Clinical Endocrinology 200665 519-523. (https://doi.org/10.1111/j.13652265.2006.02626.x)

24 Durante C, Haddy N, Baudin E, Leboulleux S, Hartl D, Travagli JP, Caillou B, Ricard M, Lumbroso JD, De Vathaire F et al. Long-term outcome of 444 patients with distant metastases from papillary and follicular thyroid carcinoma: benefits and limits of radioiodine therapy. Journal of Clinical Endocrinology and Metabolism 200691 2892-2899. (https://doi.org/10.1210/jc.2005-2838).

Received 4 February 2020

Revised version received 6 July 2020

Accepted 20 July 2020 